\title{
Differential expression of delta-like gene and protein in neuroblastoma, ganglioneuroblastoma and ganglioneuroma
}

\author{
Chih-Cheng Hsiao ${ }^{1}$, Chao-Cheng Huang ${ }^{2,3}$, Jiunn-Ming Sheen ${ }^{1}$, Ming-Hong Tai ${ }^{4}$, \\ Ching-Mei Chen $^{2}$, Lynn LH Huang ${ }^{5}$ and Jiin-Haur Chuang ${ }^{6,3}$
}

${ }^{1}$ Department of Pediatrics, Division of Hematology/Oncology, Chang Gung Memorial Hospital, Kaohsiung,
Taiwan; ${ }^{2}$ Department of Pathology, Chang Gung Memorial Hospital, Kaohsiung, Taiwan; ${ }^{3}$ The Graduate
Institute of Clinical Medical Sciences, Chang Gung University, Linko, Taiwan; ${ }^{4}$ Department of Education and
Research, Veterans General Hospital, Kaohsiung, Taiwan; ${ }^{5}$ Institute of Biotechnology, National Cheng Kung
University, Tainan, Taiwan and ${ }^{6}$ Department of Surgery, Chang Gung Memorial Hospital, Kaohsiung, Taiwan

\begin{abstract}
Neuroblastoma is an extremely malignant solid tumor in children, characterized by spontaneous differentiation and regression. An epidermal growth factor-like homeotic protein, delta-like (dlk), has been involved in differentiation of neuroblastoma cell lines, but is unknown in in vivo expression of neuroblastoma. By using in situ hybridization and immunohistochemistry, dlk mRNA and protein expression were studied in formalinfixed archival tissues from 10 patients with neuroblastoma, five with ganglioneuroblastoma, and five with ganglioneuroma. Three adrenal tissues from children died of diseases other than adrenal tumors and one from an adult with pheochromocytoma were severed as normal and disease controls. The results showed strong immunoreactive dlk staining in endothelial cells in neuroblastoma, ganglioneuroblastoma and ganglioneuroma. Dlk was detectable in mature neuromatous stroma and gangliocytes of ganglioneuroma, but not in neuroblasts of neuroblastoma and ganglioneuroblastoma, neither in gangliocytes of ganglioneuroblastoma. In contrast, dlk mRNA expression was mainly observed in the gangliocytes, but was less intense in the neuroblasts and neuromatous stroma cells. Endothelial cells were essentially devoid of dlk mRNA expression. The findings indicated that there is differential expression of $d l k$ gene and protein among neuroblastoma, ganglioneuroblastoma and ganglioneuroma. The stronger expression of dlk in gangliocytes in ganglioneuroma, in contrast to weaker or no expression in gangliocytes in ganglioneuroblastoma and neuroblasts in neuroblastoma, suggests upregulation of $d l k$ during differentiation of neuroblastoma into more benign form. Furthermore, higher dlk protein expression in the tumor endothelium than in the endothelium of normal adrenal gland implies that dlk may regulate the endothelial function in neuroblastic tumors.

Modern Pathology (2005) 18, 656-662, advance online publication, 17 December 2004; doi:10.1038/modpathol.3800335
\end{abstract}

Keywords: delta-like protein (dlk); epidermal growth factor; ganglioneuroma; ganglioneuroblastoma; neuroblastoma

Neuroblastoma cells are derived from postganglionic sympathetic neuroblasts and frequently exhibit features of neuronal differentiation. Neuroblastic tumors have the unique ability to differentiate and mature, which delineates a family composed of neuroblastoma, ganglioneuroblastoma, and ganglioneuroma. Ganglioneuroma is the most benign form, consisting of gangliocytes and mature neuromatous

Correspondence: Dr J-H Chuang, MD, Department of Surgery, Division of Pediatric Surgery, Chang Gung Memorial Hospital, 123, Ta-Pei Road, Niao Sung, Kaohsiung, Taiwan.

E-mail: jhchuang@adm.cgmh.org.tw

Received 6 July 2004; revised 7 October 2004; accepted 8 October 2004; published online 17 December 2004 stroma. Ganglioneuroblastoma is composed of both immature gangliocytes and differentiating neuroblasts and has intermediate malignant potential. Neuroblastoma is the most immature, undifferentiated, and malignant form, which may show spontaneous or induced differentiation to ganglioneuroblastoma or ganglioneuroma. The cause of trans-differentiation of one form into another is unknown, but probably involves pathways that signal the cell to differentiate. ${ }^{1}$

Delta-like protein (dlk) is a member of the epidermal growth factor (EGF)-like family, which includes proteins such as Notch, Delta, and Serrate in the control of cell differentiation. ${ }^{2}$ Structural analysis reveals that dlk contains six EGF-like 
repeats in the extracellular domains, and a short intracellular domain. ${ }^{3}$ EGF-like proteins act as receptors or as ligands that participate in cell-tocell interactions in differentiation leading to a variety of mature tissues. ${ }^{4,5}$ Dlk can be expressed as a soluble or an integral transmembrane protein, which is released to the extracellular medium by the action of specific protease. The unique structure and expression pattern of dlk suggests that dlk may play a role in the development and differentiation of a variety of organs or cell types, ${ }^{6,7}$ including adipogenesis, ${ }^{8-12}$ hematopoiesis ${ }^{13-16}$ and development of pancreas, ${ }^{17,18}$ placenta, ${ }^{19,20}$ and adrenal gland. ${ }^{21,22}$ Dlk is expressed in tumor cell lines with neuroendocrine features, including neuroblastoma, pheochromocytoma, and subsets of small cell lung carcinoma. ${ }^{20,23-26}$ The expression of dlk seems to correlate with the maturation along the chromaffin lineages. ${ }^{27}$

In this study, we characterized in vivo expression of dlk in neuroblastoma, ganglioneuroblastoma, and ganglioneuroma by using immunohistochemistry (IHC) and in situ hybridization (ISH) to bring further insights into the role of $d l k$ in differentiation and neovascularization of neuroblastoma.

\section{Materials and methods}

\section{Patients and Samples}

Formalin-fixed, paraffin-embedded specimens of neuroblastoma, ganglioneuroblastoma, and ganglioneuroma accessioned between July 1991 and December 2002 were retrieved from the archives of the Department of Pathology, Chang Gung Memorial Hospital, Kaohsiung, Taiwan. All the patients were diagnosed before the age of 19 years. These patients were classified according to the International Neuroblastoma Staging System. ${ }^{28}$

Histologic classification was according to the International Neuroblastoma Pathology Committee. $^{29}$ Based on the percentage and degree of differentiation of the neuroblastoma cells and the presence of gangliocytes, neuroblastic tumors were classified into neuroblastoma, ganglioneuroblastoma, and ganglioneuroma.

\section{Immunohistochemistry}

In all, $2-\mu \mathrm{m}$ thick tumor sections on poly-L-lysinecoated slides were dewaxed, treated with $3 \%$ hydrogen peroxide for $10 \mathrm{~min}$ to inactivate the endogenous peroxidase activity and microwaved for $10 \mathrm{~min}$ in $10 \mathrm{mM}$ citrate buffer to retrieve antigens. The sections were then incubated with goat anti-human dlk antibody (SC-8624, 1:50 dilution) (Santa Cruz Biotechnology, Santa Cruz, CA, USA) for $1 \mathrm{~h}$ at room temperature. After washing with PBS, the sections were reacted with the HRP/ Fab polymer conjugate (PicTure ${ }^{\mathrm{TM}}$-Plus kit, Zymed,
South San Francisco, CA, USA) according to the manufacturer's instructions and then incubated with DAB chromogen. The sections were finally counter stained with Gill's hematoxylin (Merck, Darmstadt, Germany). To analyze whether dlk expression in endothelial proliferation indeed was related to neurotic tumors, three specimens of adrenal gland from autopsy infants without adrenal tumor were served as the negative control. One specimen from a case of pheochromocytoma was used as a positive control. The findings of dlk expression was arbitrarily graded into three categories depending on the percentage of the cells stained: $(-)$, if less than $10 \%$ of cells were stained; $(+)$, if 10 to $50 \%$ of cells were stained; $(++)$, if $>50 \%$ of cells were stained.

\section{Nonradioactive ISH}

To detect the localization of dlk mRNA in the tumor tissue, we used a nonradioactive ISH with catalyzed reporter deposition method for signal amplification as previously described. ${ }^{30}$ For preparation of the probes, the dlk cDNA was obtained and the segment containing nucleotides 391-783 was subcloned into pGEM vector. Digoxigenin-labeled antisense riboprobes were synthesized using in vitro transcription method according to the manufacturer's instructions (Roche, Mannheim, Germany). The sense riboprobes were also synthesized by the same method to be served as the negative control in ISH. The cohort sections were prepared by cutting the paraffinembedded tissue blocks to $4 \mu \mathrm{m}$ in thickness and placing on the silane coating slides followed by deparaffinization. The procedure of deparaffinizaion and rehydration of the sections was handled under RNAse-free condition with the use of diethylpyrocarbonate water. The sections were then digested with $20 \mu \mathrm{g} / \mathrm{ml}$ proteinase $\mathrm{K}$ at $37^{\circ} \mathrm{C}$ for $25 \mathrm{~min}$, followed by acetylation in freshly prepared $0.25 \%$ acetic anhydride in $0.1 \mathrm{M}$ triethanolamine $\mathrm{pH} 8.0$ for $10 \mathrm{~min}$. The digoxigenin-labeled riboprobes were diluted in mRNA ISH solution (DAKO, Capinteria, $\mathrm{CA}$, USA) to $1 \mu \mathrm{g} / \mathrm{ml}$, denatured at $100^{\circ} \mathrm{C}$ and then added on the tissue section. The slides were placed in a humid chamber and incubated in an incubator at $50^{\circ} \mathrm{C}$ overnight for hybridization.

After hybridization, the sections were washed to remove the unbound probes and incubated with rabbit HRP-anti-digoxigenin antibody (1:150) (DAKO) in blocking buffer for $1 \mathrm{~h}$ at room temperature, followed by wash to remove unbound antibody. The signals were amplified with DAKO GenPoint kit according to the manufacturer's protocol and finally developed by adding DAB.

\section{Results}

Clinicopathological characteristics of the patients were listed in Table 1. There were 10 neuroblastoma, 
Table 1 Characteristics of 20 patients

\begin{tabular}{|c|c|c|c|c|c|}
\hline Case no. & $\begin{array}{l}\text { Age at } \\
\text { diagnosis }\end{array}$ & Gender & $\begin{array}{l}\text { INSS } \\
\text { stage }\end{array}$ & Histology & Location \\
\hline 1 & $10 \mathrm{~m}$ & $\mathrm{M}$ & IV & NB & Adrenal \\
\hline 2 & $7 y$ & $\mathrm{~F}$ & IV & NB & Adrenal \\
\hline 3 & $5 \mathrm{~m}$ & M & I & NB & Mediastinum \\
\hline 4 & $1 d$ & $\mathrm{~F}$ & IVs & NB & $\begin{array}{l}\text { Adrenal, } \\
\text { meta to liver }\end{array}$ \\
\hline 5 & $4.5 \mathrm{y}$ & $\mathrm{M}$ & III & NB & Adrenal \\
\hline 6 & $4 y$ & $\mathrm{~F}$ & IV & NB & Retroperitoneum \\
\hline 7 & $1 \mathrm{y}$ & $\mathrm{F}$ & I & NB & Mediastinum \\
\hline 8 & $3.5 \mathrm{y}$ & $\mathrm{F}$ & IV & NB & $\begin{array}{l}\text { Adrenal, } \\
\text { multiple meta }\end{array}$ \\
\hline 9 & $2.9 \mathrm{y}$ & M & IV & NB & $\begin{array}{l}\text { Adrenal, } \\
\text { meta to BM }\end{array}$ \\
\hline 10 & $10 \mathrm{~m}$ & M & II & NB & Paravertebra \\
\hline 11 & $1 \mathrm{y}$ & $\mathrm{F}$ & I & GNB & Mediastinum \\
\hline 12 & $3 \mathrm{~m}$ & $\mathrm{~F}$ & II & GNB & Mediastinum \\
\hline 13 & $8 \mathrm{~m}$ & M & III & GNB & Mediastinum \\
\hline 14 & $3.8 \mathrm{y}$ & $\mathrm{F}$ & III & GNB & Retroperitoneum \\
\hline 15 & $1 \mathrm{y}$ & M & I & GNB & Epididyma \\
\hline 16 & $11 \mathrm{y}$ & M & I & GN & Mediastinum \\
\hline 17 & $14 \mathrm{y}$ & $\mathrm{F}$ & I & GN & Mediastinum \\
\hline 18 & $2.8 \mathrm{y}$ & $\mathrm{F}$ & III & GN & Retroperitoneum \\
\hline 19 & $19 y$ & $\mathrm{M}$ & I & GN & Mediastinum \\
\hline 20 & $11 \mathrm{y}$ & $\mathrm{F}$ & I & GN & Retroperitoneum \\
\hline
\end{tabular}

$\mathrm{BM}$, bone marrow; meta, metastasis; NB, neuroblastoma; GNB, ganglioneuroblastoma; GN, ganglioneuroma; m, months; y, years.

five ganglioneuroblastoma, and five ganglioneuroma. Nine are males and 11 females. The mean age at diagnosis was 2.5 years (range, 0-7 years) for patients with neuroblastoma, 1.3 years (range, 3 months-3.8 years) for ganglioneuroblastoma, and 11.6 years (range, 2.8-19 years) for ganglioneuroma. The locations of primary tumors were adrenal gland (six), mediastinum (eight), retroperitoneum (four), paraspinal (one), and others (one). The tumors were detected in eight patients in stage I, two in stage II, four in stage III, five in stage IV, and one in stage IVs according to the International Neuroblastoma Staging System.

The results of IHC were shown in Table 2 and Figure 1. Dlk immunoreactivity was not detected in any neuroblastic cells of neuroblastoma and ganglioneuroblastoma and in immature gangliocytes of ganglioneuroblastoma, but was detected in both nuclei and cytoplasm of mature gangliocytes and neuromatous stroma of ganglioneuroma. Interestingly, the strong dlk immunoreactivity was present mainly in the cytoplasm of endothelial cells in $70 \%$ of neuroblastoma $(7 / 10), 80 \%$ of ganglioneuroblastoma $(4 / 5)$, and $80 \%$ of ganglioneuroma $(4 / 5)$. We compared dlk expression in the neuroblastoma, ganglioneuroblastoma, and ganglioneuroma from adrenal glands of normal infants and from pheochromocytoma. IHC analysis showed that the endothelial cells of pheochromocytoma were also strongly stained for dlk, similar to that observed in our neuromatous tumors. Dlk was weakly stained in the endothelial cells of normal adrenal glands and
Table 2 Immunohistochemical staining of dlk in different cell population in neuroblastoma (NB), ganglioneuroblastoma (GNB) and ganglioneuroma (GN)

\begin{tabular}{|c|c|c|c|c|}
\hline \multirow[t]{2}{*}{ Cases } & \multicolumn{3}{|c|}{$\begin{array}{l}\text { Immunohistochemical dlk } \\
\text { expression }\end{array}$} & \multirow[t]{2}{*}{$\begin{array}{l}\text { Positive rate } \\
\quad(\%)\end{array}$} \\
\hline & - & + & ++ & \\
\hline \multicolumn{5}{|l|}{ Histology } \\
\hline \multicolumn{5}{|l|}{$N B(n=10)$} \\
\hline Neuroblastic cells & 10 & 0 & 0 & 0 \\
\hline Endothelial cells & 3 & 3 & 4 & 70 \\
\hline \multicolumn{5}{|l|}{ GNB $(n=5)$} \\
\hline Neuroblastic cells & 5 & 0 & 0 & 0 \\
\hline Gangliocytes & 5 & 0 & 0 & 0 \\
\hline Endothelial cells & 1 & 0 & 4 & 80 \\
\hline \multicolumn{5}{|l|}{$G N(n=5)$} \\
\hline Neuromatous stroma & 2 & 2 & 1 & 60 \\
\hline Gangliocytes & 0 & 5 & 0 & 100 \\
\hline Endothelial cells & 1 & 2 & 2 & 80 \\
\hline
\end{tabular}

Grading of expression: -, negative; +, positive; ++, strongly positive.

was absent in the tumor cells of pheochromocytoma and in the medulla cells of normal adrenal glands.

By ISH, dlk mRNA expression was mainly observed in the gangliocytes of both ganglioneuroblastoma and ganglioneuroma, and was relatively weakly present in the neuroblastic cells and neuromatous stroma cells of neuroblastoma, ganglioneuroblastoma, and ganglioneuroma. The signals were detected in both the nuclei and cytoplasm, implying that dlk was produced in the gangliocytes, neuroblasts, and neuromatous stroma cells. However, the endothelial cells were essentially devoid of dlk mRNA expression in neuroblastoma, ganglioneuroblastoma, and rarely in ganglioneuroma (Figure 2).

\section{Discussion}

In this study, immunoreactive dlk was detectable in mature neuromatous stroma and gangliocytes of ganglioneuroma, but not in neuroblasts of neuroblastoma, ganglioneuroblastoma, neither in gangliocytes of ganglioneuroblastoma. In addition, by ISH, dlk mRNA expression is mainly observed in the gangliocytes of both ganglioneuroma and ganglioneuroblastoma, but was less intense in the neuroblastic cells of neuroblastoma, ganglioneuroblastoma, and ganglioneuroma, as well in the neuromatous stroma in ganglioneuroma. The findings indicate that there is differential expression of $d l k$ gene and protein among neuroblastoma, ganglioneuroblastoma, and ganglioneuroma. The stronger expression of dlk in gangliocytes in ganglioneuroma, in contrast to weaker or no expression in gangliocytes in ganglioneuroblastoma and neuro- 

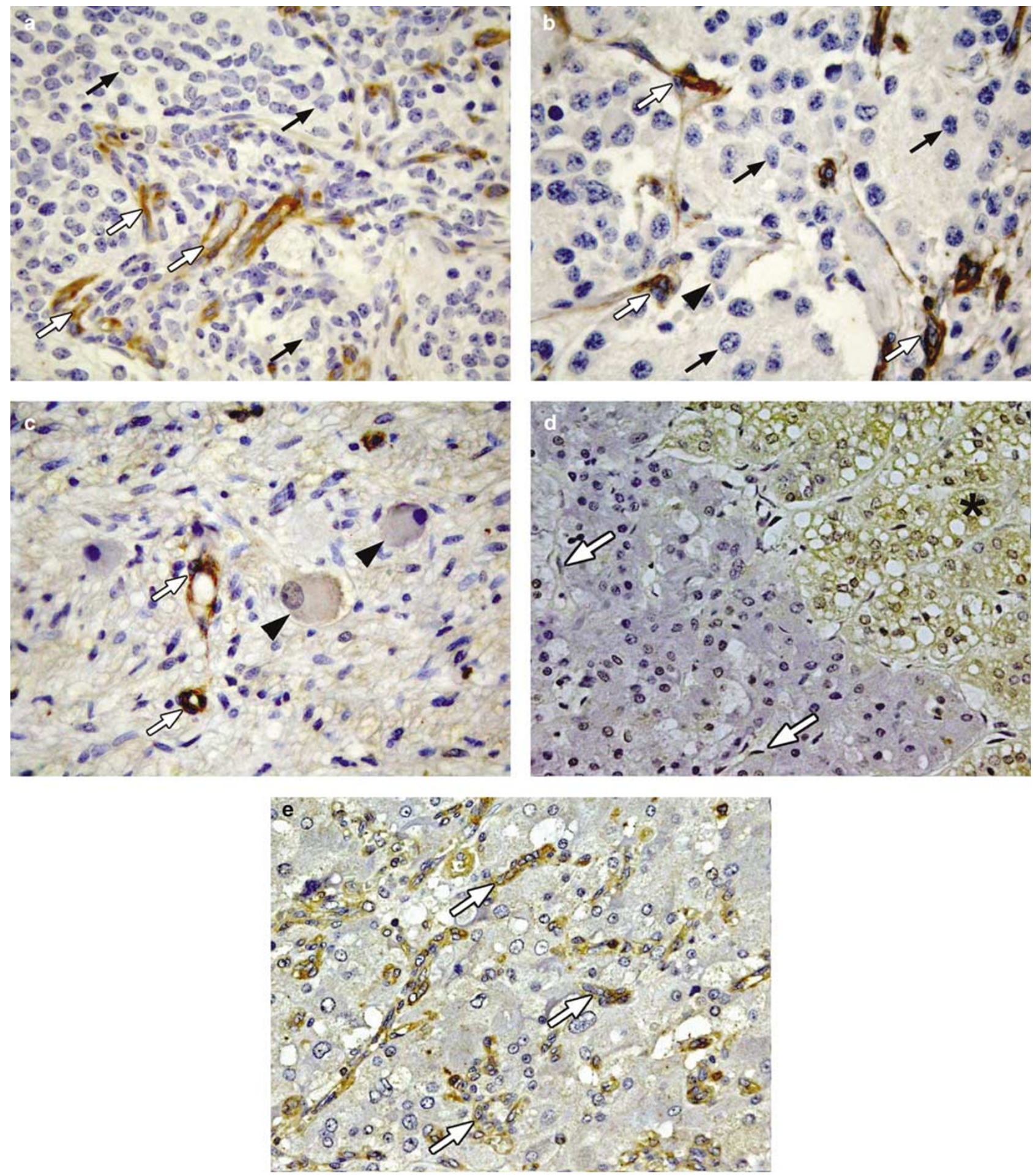

Figure 1 Immunohistochemical staining for dlk. (a) In neuroblastoma, the endothelial cells (white arrow) are strongly positive but the neuroblasts (black arrow) are negative for dlk. (b) In ganglioneuroblastoma, the staining pattern is similar to that in neuroblastoma. The immature gangliocytes (arrowhead) are also negative for dlk as well as the neuroblasts. (c) In ganglioneuroblastoma, the endothelial cells (white arrow) are still strongly positive and the mature neuromatous stroma and gangliocytes (arrowhead) are weakly positive for dlk. (d) In normal adrenal gland of a 7-month-old girl, medulla cells are negative for dlk, but the endothelial cells are weakly stained (white arrow). The cortex cells (asterisk) are strongly positive. (e) In pheochromocytoma of 24-year-old female patient, the endothelial cells (white arrow) are strongly positive but the tumor cells (black arrow) are negative for dlk (original magnification, $\times 400$ ).

blasts in neuroblastoma, suggests upregulation of dlk during differentiation of neuroblastoma into more benign form.
Our findings also demonstrated that the expression of immunoreactive dlk was present mainly in the cytoplasm of endothelial cells in neuroblastoma, 
660
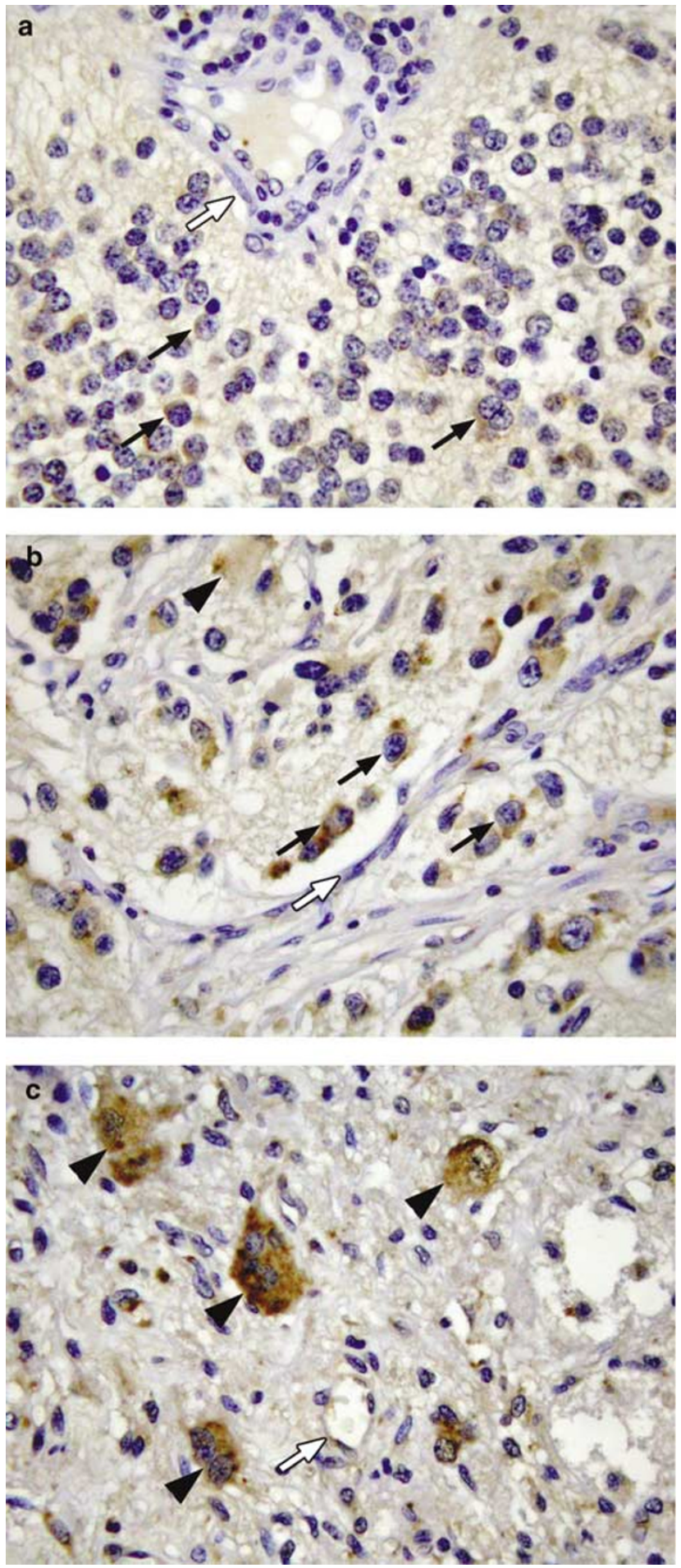

Figure 2 ISH for dlk mRNA. (a) In neuroblastoma, the endothelial cells (white arrow) are almost devoid of dlk mRNA, but the neuroblasts (black arrow) are positive for dlk mRNA expression. (b) In ganglioneuroblastoma, the dlk mRNA expression pattern is similar to that in neuroblastoma. Besides, the immature gangliocytes (arrowhead) are also positive for dlk mRNA expression. (c) In ganglioneuroma, dlk mRNA expression are rarely observed in the endothelial cells (white arrow), whereas the mature neuromatous stroma cells are weakly positive and the gangliocytes (arrowhead) are strongly positive for dlk mRNA expression (original magnification, $\times 400$ ). ganglioneuroblastoma, and ganglioneuroma, while dlk mRNA expression was not found in endothelial cells by ISH. In contrast to the distribution of dlk proteins, dlk mRNA was detected in both the cytoplasm and nuclei of neuroblastic cells and gangliocytes. The results may suggest that dlk proteins are actively produced by gangliocytes or neuroblastic cells and then transported to endothelium and neuromatous stroma. Or alternatively, lack of mRNA in nondividing endothelial cells may imply that mRNAs are short-lived and are not expressed like proteins, which are relatively more stable. However, both assumptions need further confirmation.

According to Huang et $a l^{31}$ dlk mRNA was present in hepatocytes of liver fibrosis with biliary atresia, but not in nonparenchymal cells that were strongly positive for dlk antibody in the immunohistochemical study. They suggested that dlk protein is mainly produced in hepatocytes in the liver but is transported to hepatic stellate cells via a paracrine route. This finding is consistent with ours in that dlk may act like a paracrine growth factor in regulation of endothelial function. Although the involvement of dlk in endothelial function and neovascularization remains to be elucidated, we have found an interesting dose-dependent promotion of cell proliferation and migration of human umbilical vein endothelial cells by adding the protein of the extracellular domain of dlk in vitro (unpublished data).

Dlk shows a high degree of homology with the EGF-like proteins of Drosophila and other invertebrates that are involved in the differentiation of different tissues and structures. ${ }^{32}$ In the present study, dlk protein expression is positively correlated with differentiation in the tumor cells from the most immature form, that is, neuroblastoma to the most mature form, that is, ganglioneuroma. The low abundance of dlk expression in neuroblastic cells may imply downregulation of $d l k$ in this specific population of tumor cells. These results also suggest that dlk expression may be required during the initial stages of neuroblastoma cell differentiation toward a mature phenotype.

Dlk plays an important role to trigger Notch activation and to switch between proliferation and differentiation into mature cells. ${ }^{33,34}$ Members of the Notch family attenuate the expression of proneural genes and may promote differentiation into neuronal cells. Gaetano et al studied the differentiation of primitive neuroblastoma cell lines and found that treatment with dbcAMP, an agent that induces differentiation of the chromaffin phenotype, increased the expression of dlk. Such increment in dlk expression could be inhibited by treatment with retinoic acid, which induces a neuronal phenotype. ${ }^{35,36}$ Our data coincide with these in vitro findings and demonstrate that elevated dlk expression favors the tendency toward differentiation. 
In conclusion, the results of this in vivo study indicate that $d l k$ is involved in the differentiation and the endothelial function of neuroblastic tumors. The precise mechanism or mode of action of $d l k$ in this special kind of tumor is unknown.

\section{References}

1 Shikata A, Shikata T, Sotozono Y, et al. Neuronal differentiation in human neuroblastoma cells by nerve growth factor following TrkA up-regulation by interferon- $\gamma$. Med Pediatr Oncol 2000;34:394-401.

2 Carpenter G, Zendegui JG. Epidermal growth factor, its receptor, and related proteins. Exp Cell Res 1986;164:1-10.

3 Engel J. EGF-like domains in extracellular matrix proteins: localized signals for growth and differentiation? FEBS Lett 1989;251:1-7.

4 Beatus SR, Lendahl U. Notch and neurogenesis. J Neurosci Res 1998;54:125-136.

5 Saito T, Watanabe N. Positive and negative thymocyte selection. Crit Rev Immunol 1988;18:359-370.

6 Baladron V, Ruiz-Hidalgo MJ, Gubina E, et al. Specific regions of the extracellular domain of dlk, an EGF-like homeotic protein involved in differentiation, participate in intramolecular interactions. Front Biosci 2001;6:A25-A32.

7 Laborda J. The role of the epidermal growth factor-like protein dlk in cell differentiation. Histol Histopathol 2000;15:119-129.

8 Gregoire FM, Smas CM, Sul HS. Understanding adipocyte differentiation. Physiol Rev 1998;78:783-809.

9 Smas CM, Sul HS. Characterization of Pref-1 and its inhibitory role in adipocyte differentiation. Int J Obes Relat Metab Disord 1996;20(Suppl 3):S65-S72.

10 Garces C, Ruiz-Hidalgo MJ, Bonvini E, et al. Adipocyte differentiation is modulated by secreted deltalike (dlk) variants and requires the expression of membrane-associated dlk. Differentiation 1999;64: 103-114.

11 Hansen LH, Madsen B, Teisner B, et al. Characterization of the inhibitory effect of growth hormone on primary preadipocyte differentiation. Mol Endocrinol 1998;12:1140-1149.

12 Smas CM, Sul HS. Pref-1, a protein containing EGFlike repeats, inhibits adipocyte differentiation. Cell 1993;73:725-734.

13 Ohno N, Izawa A, Hattori M, et al. dlk inhibits stem cell factor-induced colony formation of murine hematopoietic progenitors: Hes-1-independent effect. Stem Cells 2001;19:71-79.

14 Jordan CT, Van Zant G. Recent progress in identifying genes regulating hematopoietic stem cell function and fate. Curr Opin Cell Biol 1998;10:716-720.

15 Bauer SR, Ruiz-Hidalgo MJ, Rudikoff EK, et al. Modulated expression of the epidermal growth factor-like homeotic protein dlk influences stromal-cellpre-B-cell interactions, stromal cell adipogenesis, and pre-B-cell interleukin-7 requirements. Mol Cell Biol 1998;18:5247-5255.

16 Moore KA, Pytowski B, Witte L, et al. Hematopoietic activity of a stromal cell transmembrane protein containing epidermal growth factor-like repeat motifs. Proc Natl Acad Sci USA 1997;94:4011-4016.
17 Tornehave D, Jansen P, Teisner B, et al. Fetal antigen 1 (FA1) in the human pancreas: cell type expression, topological and quantitative variations during development. Anat Embryol 1993;187:335-341.

18 Tornehave D, Jansen P, Teisner B, et al. FA1 immunoreactivity in endocrine tumors and during development of the human fetal pancreas: negative correlation with glucagon expression. Histochem Cell Biol 1996; 106:535-542.

19 Jensen CH, Teisner B, Hojrup P, et al. Studies on the isolation, structural analysis and tissue localization of fetal antigen 1 and its relation to a human adrenalspecific cDNA, pG2. Hum Reprod 1993;8:635-641.

20 Laborda J, Sausville EA, Hoffman T, et al. dlk, a putative mammalian homeotic gene differentially expressed in small cell lung carcinoma and neuroendocrine tumor cell line. J Biol Chem 1993;268: 3817-3820.

21 Cooper MJ, Hutchins GM, Cohen PS, et al. Human neuroblastoma tumor cell lines correspond to the arrested differentiation of chromaffin adrenal medullary neuroblasts. Cell Growth Differ 1990;1:149-159.

22 Gaetano C, Matsumoto K, Thiele CJ. In vitro activation of distinct molecular and cellular phenotypes after induction of differentiation in a human neuroblastoma cell line. Cancer Res 1992;52:4402-4407.

23 Costaglioli P, Come C, Knoll-Gellida A, et al. The homeotic protein dlk is expressed during peripheral nerve development. FEBS Lett 2001;509:413-416.

24 Jensen CH, Kroch TN, Hojrup P, et al. Protein structure of fetal antigen 1 (FA1). A novel circulating human epidermal-growth-factor-like protein expressed in neuroendocrine tumors and its relation to the gene products of dlk and pG2. Eur J Biochem 1994;225:83-92.

25 Krogh TN, Bachmann E, Teisner B, et al. Glycosylation analysis and protein structure determination of murine fetal antigen 1 (mFA1) - the circulating gene product of the delta-like protein (dlk), preadipocyte factor 1 (Pref1 ) and stromal-cell-derived protein 1 (SCP-1) cDNAs. Eur J Biochem 1997;244:334-342.

26 Ruiz-Hidalgo MJ, Gubina E, Tull L, et al. dlk modulates mitogen-activated protein kinase signaling to allow or prevent differentiation. Exp Cell Res 2002;274: 178-188.

27 Van Limpt VA, Chan AJ, Van Sluis PG, et al. High delta-like 1 expression in a subset of neuroblastoma cell lines corresponds to a differentiated chromaffin cell type. Int J Cancer 2003;105:61-69.

28 Brodeur GM, Pritchard J, Berthold F, et al. Revisions of the international criteria for neuroblastoma diagnosis, staging and response to treatment. Prog Clin Biol Res 1994;385:363-369.

29 Shimada H, Ambros IM, Dehner LP, et al. The International Neuroblastoma Pathology Classification (the Shimada system). Cancer 1999;86:364-372.

30 Liu RT, Huang CC, You HL, et al. Overexpression of tumor susceptibility gene TSG101 in human papillary thyroid carcinomas. Oncogene 2002;21:4830-4837.

31 Huang CC, Chuang JH, Huang LL, et al. The human delta-like 1 homologue is implicated in the progression of liver fibrosis in biliary atresia. J Pathol 2004; 202:172-179.

32 Baladron V, Ruiz-Hidalgo MJ, Bonvini E, et al. The EGF-like homeotic protein dlk affects cell growth and interacts with growth-modulating molecules in the yeast two-hybrid system. Biochem Biophys Res Commun 2002;291:193-204. 
33 Seugnet L, Simpson P, Haenlin M. Transcriptional regulation of Notch and Delta: requirement for neuroblast segregation in Drosophila. Development 1997; 124:2015-2025.

34 Limpt VV, Chan A, Caron H, et al. SAGE analysis of neuroblastoma reveals a high expression of the human homologue of the Drosophila delta gene. Med Pediatr Oncol 2000;35:554-558.
35 Gaetano C, Matsumoto K, Thiele CJ. In vitro activation of distinct molecular and cellular phenotypes after induction of differentiation in a human neuroblastoma cell line. Cancer Res 1992;52:4402-4407.

36 Gaetano C, Manni I, Bossi G, et al. Retinoic acid and cAMP differentially regulate human chromogranin A promoter activity during differentiation of neuroblastoma cells. Eur J Cancer 1995;31A:447-452. 